\title{
Effects of Intensive Blood Pressure Lowering on Cardiovascular and Renal Outcomes: A Systematic Review and Meta-Analysis
}

\author{
Jicheng Lv ${ }^{1,2}$, Bruce Neal ${ }^{1}$, Parya Ehteshami ${ }^{1}$, Toshiharu Ninomiya ${ }^{4}$, Mark Woodward ${ }^{1,3}$, \\ Anthony Rodgers ${ }^{1}$, Haiyan Wang ${ }^{2}$, Stephen MacMahon ${ }^{1}$, Fiona Turnbull' ${ }^{1}$, Graham Hillis ${ }^{1}$, \\ John Chalmers ${ }^{1}$, Vlado Perkovic ${ }^{1 *}$
}

1 The George Institute for Global Health, The University of Sydney, Sydney, Australia, 2 Renal Division, Department of Medicine, Peking University First Hospital, Beijing, China, 3 Department of Epidemiology, Johns Hopkins University, Baltimore, Maryland, United States of America, 4 Department of Medicine and Clinical Science Graduate School of Medical Sciences, Kyushu University, Japan

\begin{abstract}
Background: Guidelines recommend intensive blood pressure (BP) lowering in patients at high risk. While placebocontrolled trials have demonstrated $22 \%$ reductions in coronary heart disease (CHD) and stroke associated with a $10-\mathrm{mmHg}$ difference in systolic BP, it is unclear if more intensive BP lowering strategies are associated with greater reductions in risk of CHD and stroke. We did a systematic review to assess the effects of intensive BP lowering on vascular, eye, and renal outcomes.

Methods and Findings: We systematically searched Medline, Embase, and the Cochrane Library for trials published between 1950 and July 2011. We included trials that randomly assigned individuals to different target BP levels. We identified 15 trials including a total of 37,348 participants. On average there was a $7.5 / 4.5-\mathrm{mmHg}$ BP difference. Intensive BP lowering achieved relative risk (RR) reductions of $11 \%$ for major cardiovascular events $(95 \% \mathrm{Cl} 1 \%-21 \%), 13 \%$ for myocardial infarction $(0 \%-25 \%), 24 \%$ for stroke $(8 \%-37 \%)$, and $11 \%$ for end stage kidney disease $(3 \%-18 \%)$. Intensive BP lowering regimens also produced a $10 \%$ reduction in the risk of albuminuria (4\%-16\%), and a trend towards benefit for retinopathy $(19 \%, 0 \%-34 \%, p=0.051)$ in patients with diabetes. There was no clear effect on cardiovascular or noncardiovascular death Intensive BP lowering was well tolerated; with serious adverse events uncommon and not significantly increased, except for hypotension (RR 4.16, 95\% Cl 2.25 to 7.70$)$, which occurred infrequently (0.4\% per 100 person-years).
\end{abstract}

Conclusions: Intensive BP lowering regimens provided greater vascular protection than standard regimens that was proportional to the achieved difference in systolic BP, but did not have any clear impact on the risk of death or serious adverse events. Further trials are required to more clearly define the risks and benefits of BP targets below those currently recommended, given the benefits suggested by the currently available data.

Please see later in the article for the Editors' Summary.

Citation: Lv J, Neal B, Ehteshami P, Ninomiya T, Woodward M, et al. (2012) Effects of Intensive Blood Pressure Lowering on Cardiovascular and Renal Outcomes: A Systematic Review and Meta-Analysis. PLoS Med 9(8): e1001293. doi:10.1371/journal.pmed.1001293

Academic Editor: Mark J. Caulfield, Barts and The London School of Medicine and Dentistry, United Kingdom

Received October 7, 2011; Accepted July 6, 2012; Published August 21, 2012

Copyright: (c) 2012 Lv et al. This is an open-access article distributed under the terms of the Creative Commons Attribution License, which permits unrestricted use, distribution, and reproduction in any medium, provided the original author and source are credited.

Funding: JL was supported by an Amgen Renal Research Fellowship. VP was supported by an Australian Heart Foundation Career Development Award. The funders had no role in study design, data collection and analysis, decision to publish, or preparation of the manuscript.

Competing Interests: JL has received grant support from Pfizer for hypertension research. VP, MW, SM, and JC have received honoraria from Servier for scientific presentations relating to blood pressure. SM and JC were principal investigators on ADVANCE, a blood pressure lowering trial funded by Servier and the Australian National Health and Medical Research Council. BN has received BP-related research support from Servier, and honoraria for scientific presentations related to blood pressure from Novartis, Tanabe, and Servier. AR has received an unrestricted grant from Dr Reddy's Laboratories for a trial that includes blood pressure-lowering agents. $\mathrm{PE}, \mathrm{FT}, \mathrm{TN}, \mathrm{HW}$, and $\mathrm{GH}$ declare they have no competing interests.

Abbreviations: BP, blood pressure; CHD, coronary heart disease; CKD, chronic kidney disease; ESKD, end stage kidney disease; RR, relative risk

*E-mail: vperkovic@george.org.au 


\section{Introduction}

Cohort studies show continuous positive associations of blood pressure $(\mathrm{BP})$ with cardiovascular risk with no evidence of a threshold at BP levels down to $110 / 70 \mathrm{mmHg}$ [1-3]. Large-scale placebo-controlled randomised trials of BP lowering have achieved reductions in risk of $22 \%$ for coronary heart disease (CHD) and $41 \%$ for stroke for every $10 \mathrm{mmHg}$ lower $\mathrm{BP}$ achieved, that the risk reduction correlates almost exactly with that anticipated from epidemiological studies [4,5]. In addition, in trials of BP lowering versus control, the greater $\mathrm{BP}$ reductions achieved by combination treatment have produced greater risk reductions than those obtained for monotherapy [4]. Finally, in trials comparing different $\mathrm{BP}$ lowering agents, the trials with larger BP differences have also resulted in greater differences in effects on clinical outcomes [5].

As trial evidence has accumulated, the BP targets recommended by guideline groups have been progressively lowered and intensive BP lowering is now widely advocated for individuals at high cardiovascular risk [6-10]. These recommendations are, however, still debated in recent national guidelines [11-14], due in part to some observational analyses that have reported associations of low BP with increased coronary disease risk. However, it is not certain whether this is causal or represents the effects of preclinical disease both lowering BP and independently increasing risk. A 2003 systematic overview that included five trials and about 22,000 individuals concluded that more intensive BP lowering provided significantly greater cardiovascular protection but did not address a key question about the effects of targeting different BP levels [5]. More recently, a Cochrane review using different trial inclusion criteria reported no greater benefit for intensive regimens targeting BP levels of $<135 / 85 \mathrm{mmHg}$ compared to standard BP targets [15].

The completion, in the last few years, of three large new trials evaluating the effects of different intensities of BP lowering on cardiovascular outcomes provides an opportunity to re-assess the evidence for lower BP targets [16-18]. In this systematic review, we sought to synthesize all the available clinical trial data and better define the balance of risks and benefits associated with different intensities of BP lowering.

\section{Methods}

\section{Data Sources and Searches}

We performed a systematic review of the literature in line with the approach recommended by the PRISMA statement for the conduct of meta-analyses of intervention studies (Text S1) [19]. Relevant studies were identified by searching the following data sources: MEDLINE via Ovid (from 1950 through July 2011), EMBASE (from 1966 through July 2011), and the Cochrane Library database, using relevant text words and medical subject headings that included all spellings of antihypertensive agents, target $\mathrm{BP}$, intensive $\mathrm{BP}$ treatment, intensive $\mathrm{BP}$ control, strict $\mathrm{BP}$ treatment, strict $\mathrm{BP}$ control, tight $\mathrm{BP}$ treatment, and tight $\mathrm{BP}$ control (see Text S2). The search was limited to randomized controlled trials with at least 6 mo follow-up, but without age or language restriction. Reference lists from identified trials and review articles were manually scanned to identify any other relevant studies. The ClinicalTrials.gov website was also searched for randomized trials that were registered as completed but not yet published.

\section{Study Selection}

The literature search, data extraction, and quality assessment were conducted independently by two authors using a standard- ized approach (JL and PE). All completed randomized controlled trials that compared more versus less intensive BP targets with pharmacological BP lowering agents were eligible for inclusion, including those that included participants with hypertension, high vascular/renal risk, or both.

\section{Data Extraction and Quality Assessment}

Published reports were obtained for each trial and standard information was extracted into a spreadsheet. The data sought included baseline patient characteristics (age, gender, mean systolic and diastolic BP levels, history of diabetes, history of hypertension, and chronic kidney disease [CKD]), BP control target in each arm, BP lowering agents, follow-up duration, mean reduction of systolic and diastolic $\mathrm{BP}$ during the trial, outcome events, and adverse events. Study quality was judged by the proper conduct of randomization, concealment of treatment allocation, similarity of treatment groups at baseline, the provision of a description of the eligibility criteria, completeness of follow-up, and use of intention-to-treat analysis. The Cochrane Collaboration's tool was used for assessing risk of bias. Any disagreement in abstracted data was adjudicated by a third reviewer (VP).

\section{Outcomes}

The primary outcome was major cardiovascular events defined as a composite of myocardial infarction, stroke, heart failure, and cardiovascular death. Secondary outcomes were each individual component of the composite primary outcome, all-cause mortality, end stage kidney disease (ESKD), and adverse outcomes. Progression of albuminuria (defined as new onset of micro-/ macro-albuminuria or microalbuminuria to macroalbuminuria) and retinopathy (retinopathy progression $\geq 2$ steps) were also recorded for trials done in patients with diabetes.

\section{Data Synthesis and Analysis}

Individual patient data (IPD) were not available for the studies in this analysis so tabular data were used. Individual study relative risk (RR) ratios and 95\% CIs were calculated for each outcome before pooling. Where continuous scales of measurement were used to assess the effects of treatment (BP), then the mean difference (MD) was used. Summary estimates of RR ratios or MD were obtained using a random effects model. The percentage of variability across studies attributable to heterogeneity beyond chance was estimated using the $I^{2}$ statistic [20]. Potential publication bias was assessed using the Egger test and represented graphically using Begg funnel plots of the natural log of the RR versus its standard error [21]. Evidence for heterogeneity in estimates of treatment effect attributable to the baseline characteristics of the trials was explored by comparing summary results obtained from subsets of studies grouped by number of patients, cardiovascular event rate, age, diabetes, BP target, and BP level at baseline. A two-sided $p$-value less than 0.05 was considered statistically significant and statistical analyses were performed using STATA version 10.1 (Stata).

\section{Results}

\section{Search Results and Characteristics of Included Studies}

The literature search yielded 1,650 articles of which 67 were reviewed in full text and from which 15 randomized controlled trials reported in 17 publications were identified (Figure 1) [1618,22-35]. These trials provided information on a total of 37,348 patients among whom 1,984 major cardiovascular events were reported from ten studies, 1,584 deaths from 15 studies, and 941 
ESKD events from eight studies. All the trials were open designs with few patients lost to follow-up $(0 \%-4.9 \%)$. Mean study followup duration ranged from 1.6 to $12.2 \mathrm{y}$. The reported trial quality varied substantially (Table $\mathrm{S} 1$ ).

Among the 15 trials, five $(n=6,960)$ enrolled only patients with diabetes $[16,23,25,26,31]$ and six specifically recruited participants with CKD $(n=2,734)$ (Table 1) $[22,29,30,33-35]$. One of the studies was done in children with CKD and hypertension $(n=385$, with mean age 11.5 y) [33].

Two trials $(n=609)$ recruited diabetic patients without hypertension with mean baseline BP 136/84 and 126/84 mmHg [26,31]. The other 12 trials $(n=36,664)$ recruited patients with hypertension [16-18,22-30,32,35]. The mean baseline BP levels in the trials of adults were between $131 / 80$ and $172 / 105 \mathrm{mmHg}$ and $109 / 64$ in the trial done in children.

The BP targets varied substantially between trials. The three most conservative trials sought to meet or better intensive group targets of $140-150 \mathrm{mmHg}$ systolic and $85-90 \mathrm{mmHg}$ diastolic $[18,23,32]$, while the most aggressive studies had systolic BP targets that were $20-30 \mathrm{mmHg}$ below these levels [16,17,30,34]. Four trials had diastolic BP targets below $80 \mathrm{mmHg}$ [24-26,31]. Across all trials, the weighted mean follow-up difference in BP between the more versus less intensively treated groups was $7.5 \mathrm{mmHg}$ for systolic BP and $4.5 \mathrm{mmHg}$ for diastolic BP.

\section{Effects of Intensive BP Lowering Regimens}

Major cardiovascular events. Data regarding the effects of intensive BP regimens on major cardiovascular events were available from ten trials including 35,842 participants and 1,984 cardiovascular events (Figure 2a). Overall, more intensive BP lowering regimens produced an $11 \%$ (RR 0.89, 95\% CI 0.79 $0.99, p=0.036)$ reduction in the risk of major cardiovascular events compared to less intensive regimens with no evidence of heterogeneity in the magnitude of the effect across the included studies $\left(I^{2}=28.2 \%, p=0.185\right)$.

Gause-specific vascular outcomes. Myocardial infarction was reported by nine trials including 34,748 participants among whom 756 events were observed (Figure 2b). More intensive BP lowering therapy reduced the risk of myocardial infarction by $13 \%$ (RR 0.87, 95\% CI 0.75-1.00, $p=0.049)$. There were ten trials $(35,842$ participants) that reported 726 stroke events and nine trials $(32,582$ participants) reported 427 occurrences of heart failure. More intensive BP regimens were associated with a $24 \%$ (RR 0.76 , 95\% CI $0.63-$ $0.92, p=0.004$ ) lower risk of stroke (Figure 2c), but there was no clearly apparent beneficial effect for heart failure (RR 0.93, 95\% CI $0.73-1.20, p=0.577$ ) (Figure 3a). As illustrated in Table 2, the magnitudes of the risk reductions observed for stroke $(24 \%, 95 \%$ CI 8\%-37\%) and CHD (13\%, 95\% 0\%-25\%) in this meta-analysis were directly comparable to those anticipated from large cohort studies
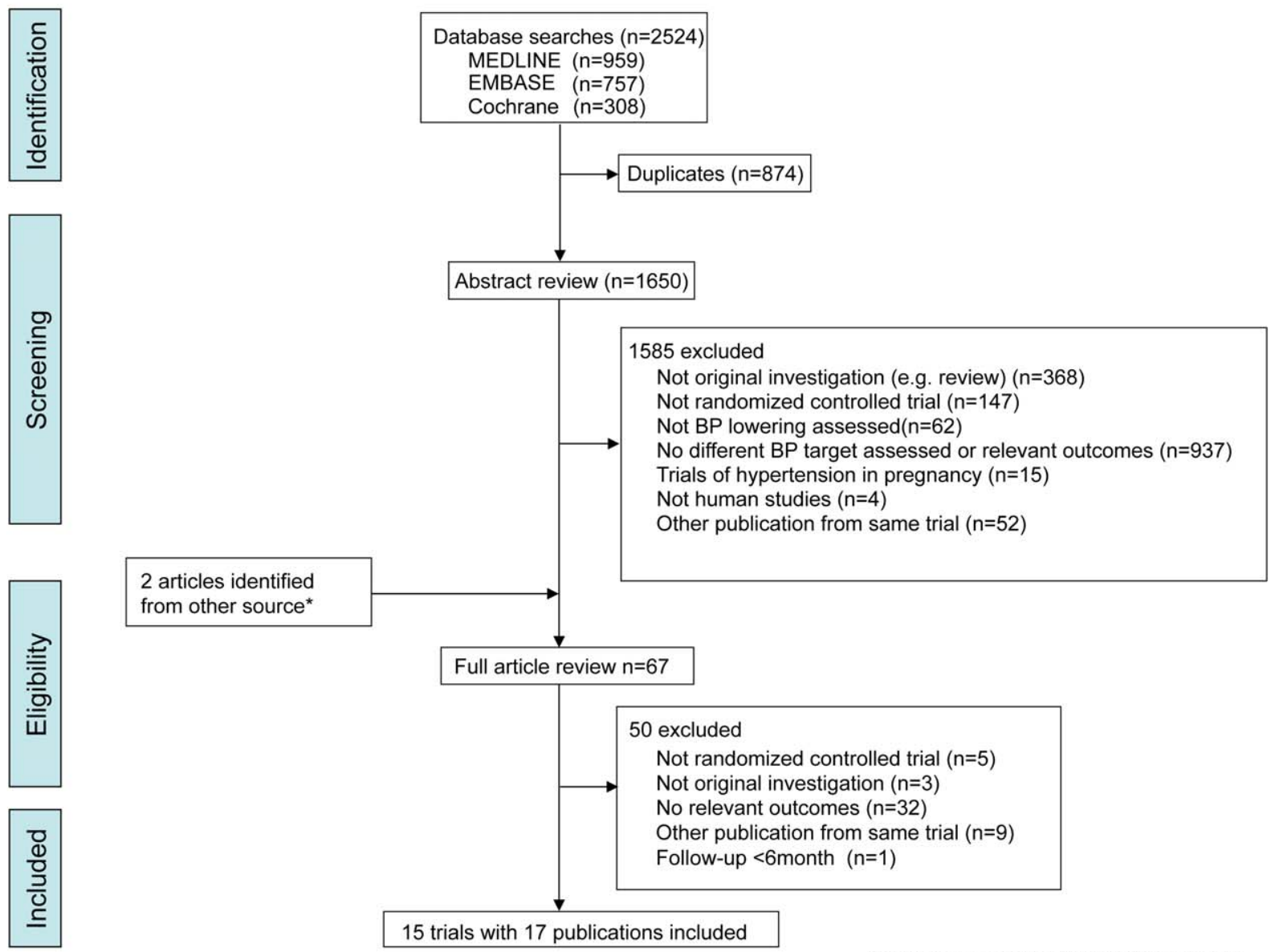

*searching on www.clinicaltrials.gov and references

Figure 1. Identification process for eligible studies. doi:10.1371/journal.pmed.1001293.g001 


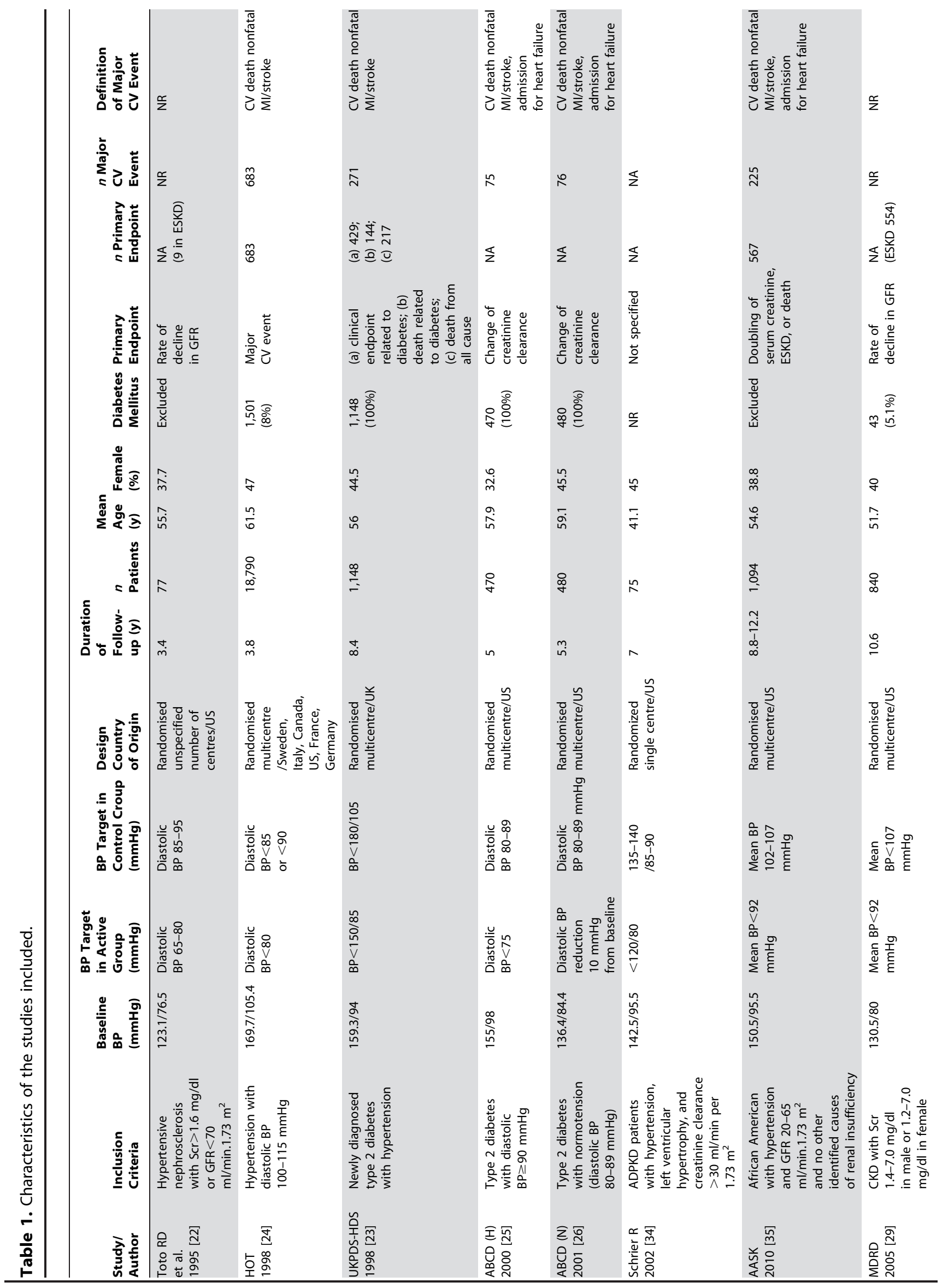




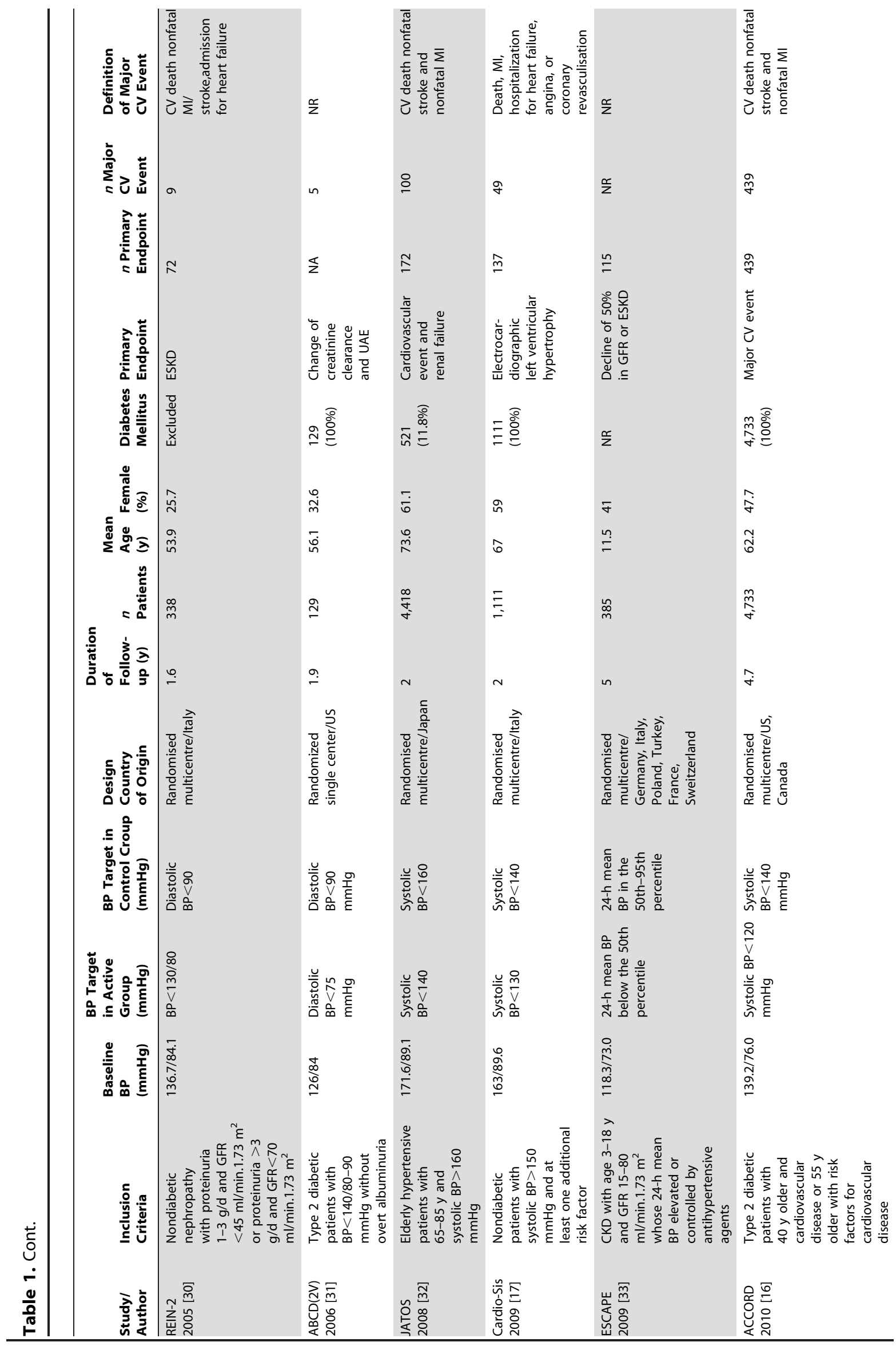




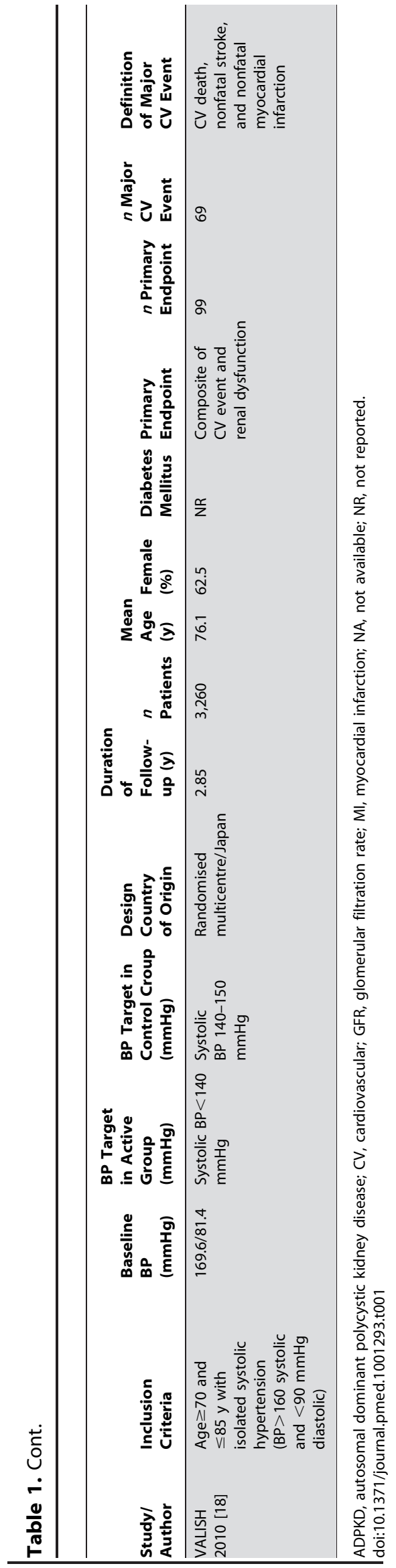

Table 2. Comparison of expected and observed effects of a 7.5-mmHg systolic blood pressure difference on coronary heart disease, stroke, and heart failure.

\begin{tabular}{lll}
\hline Relative Risk Reduction & CHD & Stroke \\
\hline Expected $^{\text {a }}$ from cohort studies & $19 \%$ & $27 \%$ \\
Observed $^{\mathrm{a}}$ in trials of BP lowering versus control & $17 \%$ & $33 \%$ \\
Observed in trials of more versus less BP lowering & $13 \%$ & $24 \%$ \\
\hline
\end{tabular}

${ }^{\text {a }}$ The associations observed in cohort studies [2] and the reductions shown in trials of BP lowering versus control [4] are shown, standardized to the 7.5$\mathrm{mmHg}$ systolic difference seen in the current meta-analysis (e.g., previous trials showed a RR for stroke of 0.59 with a $10 \mathrm{mmHg}$ systolic reduction, so one would expect a $33 \%$ reduction for $7.5 \mathrm{mmHg}$ lower systolic, as $0.59^{7.5 / 10}=0.67$ ). doi:10.1371/journal.pmed.1001293.t002

(stroke 27\% and CHD 19\% with a 7.5-mmHg systolic BP difference) [2]. The effects were also consistent with the observed effects of a meta-analysis of trials comparing BP lowering agents against control that standardized to a $7.5-\mathrm{mmHg}$ systolic BP difference between randomized groups (stroke 33\% and CHD 17\%) [4].

Fatal events. There was no clear effect of more intensive BP lowering on the risk of cardiovascular death (RR 1.00, 95\% CI $0.82-1.22, p=0.979)$ (Figure 3b), noncardiovascular death ( $R R$ $0.97,95 \%$ CI $0.84-1.11, p=0.621$ ), or all-cause death (RR 1.00, 95\% CI 0.91 to $1.10, p=0.995)$ as compared with less intensive BP control, with CIs that were compatible with modest effects in either direction.

End stage kidney disease. Eight trials including 8,690 participants recorded 941 ESKD outcomes. Compared to less intensive BP lowering, a more intensive regimen reduced the risk of ESKD by $11 \%$ (RR $0.89,95 \%$ CI $0.82-0.97, p=0.009$ ) without evidence of heterogeneity $\left(I^{2}=0 \%, p=0.444\right)$ (Figure 3c).

Microvascular events in diabetes. Three trials reported data on progression of albuminuria (5,224 participants and 1,924 events) and more intensive $\mathrm{BP}$ control reduced the risk of albuminuria progression by $10 \%$ (RR $0.90,95 \%$ CI $0.84-0.96, p=0.004$ ) with no evidence of heterogeneity $\left(I^{2}=0.0 \%, p=0.649\right)$ (Figure 4). Progression of retinopathy was reported by four trials with 2,665 participants and 693 events. There was a borderline significant reduction in retinopathy with more intensive BP lowering (RR 0.81, 95\% CI $0.66-1.00, p=0.051$ ) but substantial heterogeneity in the magnitude of the effect across the included studies $\left(I^{2}=65.5 \%, p=0.033\right)$ (Figure 4) mostly attributable to the ACCORD result. A sensitivity analysis excluding ACCORD resulted in a risk reduction of 25\% (RR $0.75,95 \%$ CI $0.65-0.86, p<0.001)$ with a much reduced $I^{2}$ value of $18.1 \%$. Of note, there were significant imbalances in a number of the baseline characteristics between randomized arms in this substudy of ACCORD [4].

\section{Potential Harms of Treatment}

Data on adverse outcomes potentially associated with treatment were collected from the trials but were inconsistently reported (Table 3). Five trials reported data on severe adverse events (SAEs) (9,827 participants and 564 events) [16-18,30,33] and four trials on total adverse events (AEs) (9,174 participants and 1,877 events) $[17,18,33,36]$ showing no clear effect of more intensive BP lowering compared to less intensive BP lowering on SAEs (RR 1.19, 0.88$1.61, p=0.250)$ or AEs (RR 0.99, 0.92-1.08, $p=0.844)$. Four trials $[16,17,29,33]$ reported hypotension outcomes $(5,118$ participants, with 76 versus 16 events) with more intensive BP control greatly increasing the risk of hypotension (RR 4.16, 95\% CI 2.25-7.70, $p<0.001)$ and showing an adverse trend towards severe hypotension 


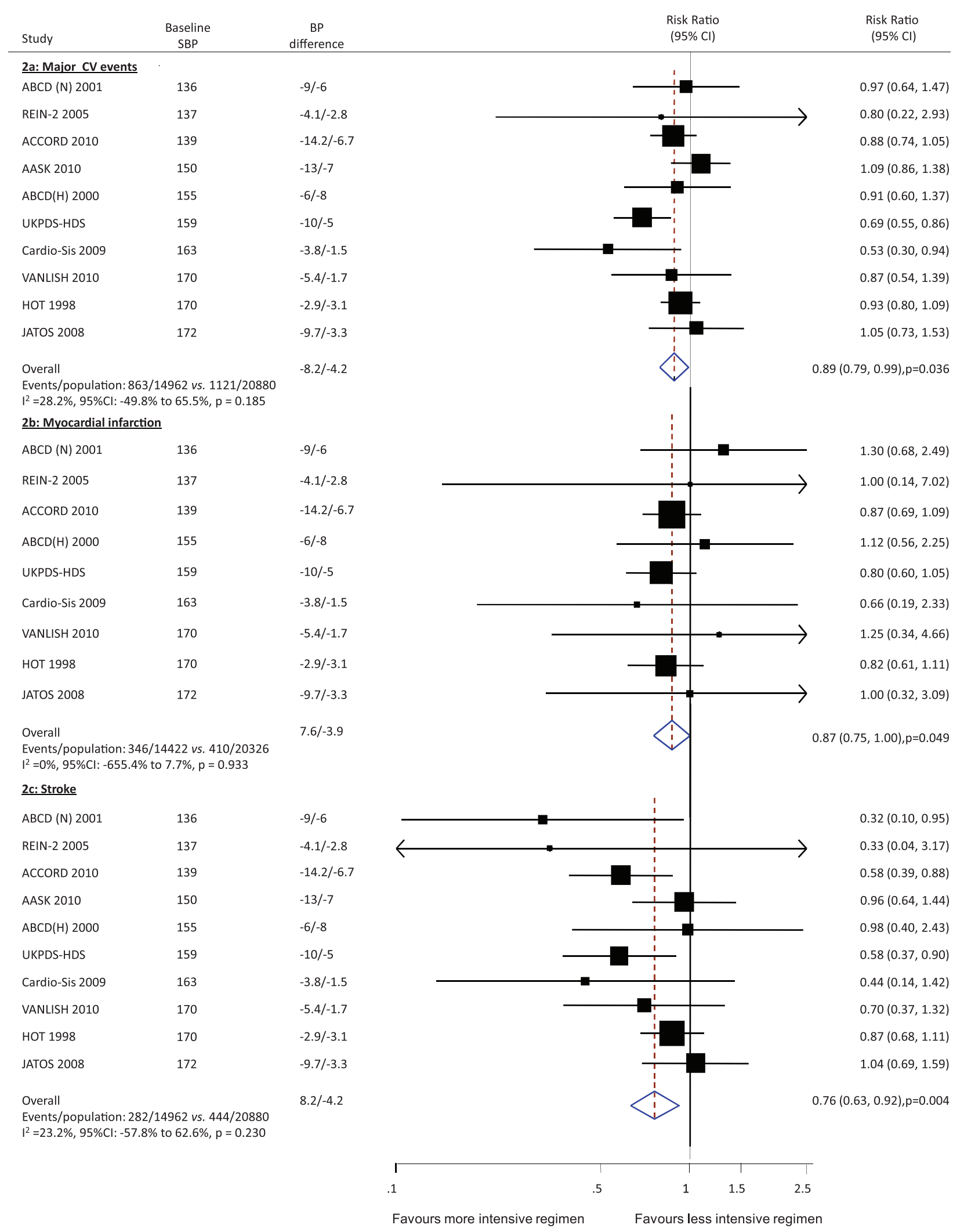

BP: blood pressure, SBP: systolic blood pressure $(\mathrm{mmHg})$

Figure 2. Effect of intensive BP lowering on risk of major cardiovascular events (a), myocardial infarction (b), and stroke (c). Boxes and horizontal lines represent RR and $95 \% \mathrm{Cl}$ for each trial. Size of boxes is proportional to weight of that trial result. Diamonds represent the $95 \% \mathrm{Cl}$ for pooled estimates of effect and are centered on pooled RR. doi:10.1371/journal.pmed.1001293.g002 


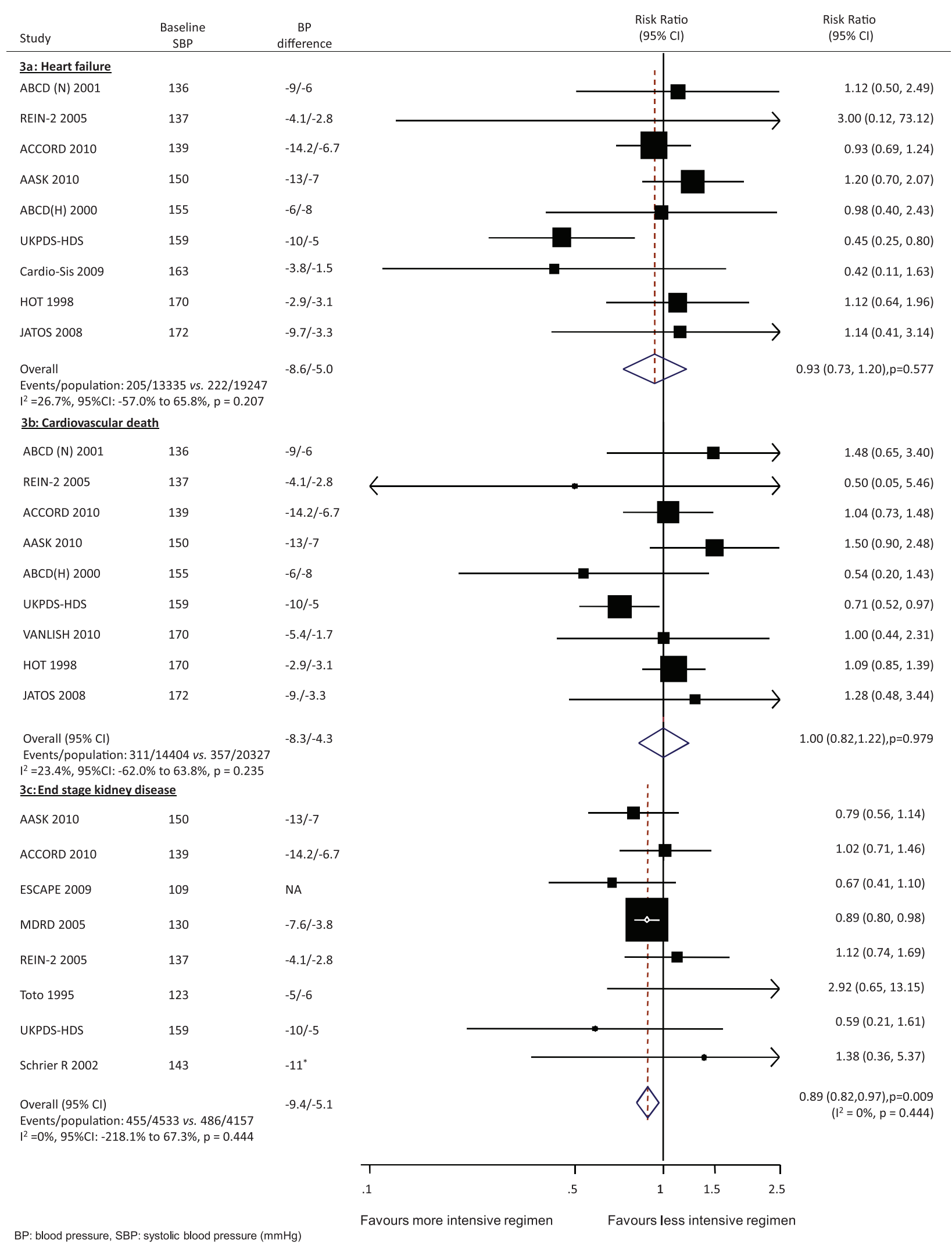

${ }^{*}$ Mean arterial pressure difference

Figure 3. Effect of intensive BP lowering on risk of heart failure (a), cardiovascular death (b), and end stage kidney disease (c). Boxes and horizontal lines represent RR and $95 \% \mathrm{Cl}$ for each trial. Size of boxes is proportional to weight of that trial result. Diamonds represent the $95 \% \mathrm{Cl}$ for pooled estimates of effect and are centered on pooled RR.

doi:10.1371/journal.pmed.1001293.g003 


\begin{tabular}{|c|c|c|c|c|}
\hline Study & $\begin{array}{l}\text { Baseline } \\
\text { SBP }\end{array}$ & $\begin{array}{c}\text { BP } \\
\text { difference }\end{array}$ & $\begin{array}{c}\text { Risk Ratio } \\
(95 \% \mathrm{Cl})\end{array}$ & $\begin{array}{c}\text { Risk Ratio } \\
(95 \% \mathrm{Cl})\end{array}$ \\
\hline \multicolumn{5}{|c|}{ Retinopathy progression } \\
\hline ABCD (N) 2001 & 136 & $-9 /-6$ & & $0.74(0.59,0.93)$ \\
\hline ACCORD 2010 & 139 & $-14.2 /-6.7$ & & $1.18(0.84,1.66)$ \\
\hline $\mathrm{ABCD}(\mathrm{H}) 2000$ & 155 & $-6 /-8$ & & $0.85(0.68,1.04)$ \\
\hline UKPDS-HDS 1998 & 159 & $-10 /-5$ & & $0.66(0.53,0.83)$ \\
\hline $\begin{array}{l}\text { Overall } \\
\text { Events/population: } 3\end{array}$ & 1421 versu & $1244^{-11.2 /-6.3}$ & & $\begin{array}{r}0.81(0.66,1.00), p=0.051 \\
\left(\left.\right|^{2}=65.5 \%, p=0.033\right)\end{array}$ \\
\hline Excluding ACCORD & & & & $\begin{array}{r}0.75(0.65,0.86), p<0.001 \\
\quad\left(\left.\right|^{2}=18.1 \%, p=0.295\right)\end{array}$ \\
\hline \multicolumn{5}{|c|}{ Albuminuria progression } \\
\hline ACCORD 2010 & 139 & $-14.2 /-6.7$ & $\rightarrow$ & $0.90(0.83,0.97)$ \\
\hline ABCD (H) 2001 & 155 & $-6 /-8$ & & $1.07(0.73,1.59)$ \\
\hline UKPDS-HDS 1998 & 159 & $-10 /-5$ & 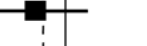 & $0.87(0.66,1.15)$ \\
\hline Overall & & $-10.1 /-6.4$ & 1 & $0.90(0.84,0.96) p=0.004$ \\
\hline \multicolumn{3}{|c|}{ Events/population: $926 / 2661$ versus $998 / 2563$} & V & $\left(I^{2}=0.0 \%, p=0.649\right)$ \\
\hline & & 1 & & \\
\hline & & .1 & 1 & \\
\hline
\end{tabular}

BP: blood pressure, SBP: systolic blood pressure $(\mathrm{mmHg})$

Figure 4. Effect of intensive BP lowering on the risk of microvascular outcomes in diabetes. doi:10.1371/journal.pmed.1001293.g004

(RR 2.19, 95\% CI 0.03-164.77, $p=0.723$ ) although the annual rate of severe hypotension was very low $(0 \%-0.15 \%)$ [16,33]. More intensive $\mathrm{BP}$ control did not clearly increase the risk of dizziness (three trials, 6,629 participants, and 413 events; RR 1.15, 95\% CI $0.95-1.38, p=0.148)[16,17,33]$. Finally, there was no clear difference detected in the rate of drug discontinuation between the more intensive and less intensive treated groups in the four trials that reported data (9,874 participants, 340 events; RR 0.96, 95\% CI $0.79-1.16)[16,17,32,33]$.

\section{Effects in Trial Subgroups}

There was no evidence that the observed effects of more intensive BP lowering regimens differed amongst trial subgroups defined according to a broad range of baseline characteristics ( $p$ for heterogeneity all $p>0.05$ ) (Figure 5). In particular, there was no clear evidence that the benefits of more intensive BP lowering varied by the starting mean baseline $\mathrm{BP}$ of the trial participants or the absolute level of the systolic or diastolic target set for the intensive group. Univariate meta-regression of intensive BP lowering on major cardiovascular outcomes according to the baseline characteristics also showed no evidence of heterogeneity (Table 4).
Formal statistical testing showed no obvious evidence of publication bias for the outcome of major vascular outcomes $(p>0.05)$; however, the power to detect publication bias was limited as on only eight to ten studies were available for each comparison (Figure $\mathrm{S} 1$ ).

\section{Discussion}

This meta-analysis, including more than 37,000 individuals amongst whom over 1,900 major vascular events were recorded, demonstrates a clear vascular benefit for more intensive BP lowering regimens aiming for lower BP targets. Major cardiovascular events were reduced by $11 \%$ and serious renal outcomes by $11 \%$ with specific benefit for a broad range of cardiovascular and renal outcomes, including myocardial infarction, stroke, albuminuria, and ESKD. However, there was no evidence to suggest that intensive BP treatment reduced or increased the risk of cardiovascular or noncardiovascular mortality. To the extent that it was possible to explore them, the observed beneficial effects did not appear to be attenuated by any characteristics of the patients involved or the BP regimens tested. Some adverse effects were more common in the intensively treated groups, but there was no suggestion that more intensive regimens were likely to result in net 
Table 3. Adverse events between more intensive and less intensive BP lowering regimen.

\begin{tabular}{|c|c|c|c|c|c|}
\hline Adverse Event & Study & Participants & $\begin{array}{l}\text { Events Rate* (More/Less } \\
\text { Intensive) }\end{array}$ & RR (95\% Cl) & $p$-Value \\
\hline Total Severe AEs $[16-18,30,33]$ & 5 & 9,827 & $309(1.7) / 255(1.4)$ & $1.19(0.88-1.61)$ & 0.250 \\
\hline Total AEs $[17,18,33,36]$ & 4 & 9,174 & $934(8.4) / 943(8.5)$ & $0.99(0.92-1.08)$ & 0.844 \\
\hline Discontinue medication $[16,17,32,33]$ & 4 & 9,874 & $179(1.1) / 161(1.0)$ & $0.96(0.79-1.16)$ & 0.663 \\
\hline \multicolumn{6}{|c|}{ Total AEs associated with BP medication } \\
\hline Hypotension $[16,17,29,33]$ & 4 & 14,138 & $76(0.4) / 16(0.08)$ & $4.16(2.25-7.70)$ & $<0.001$ \\
\hline Dizziness $[16,17,33]$ & 3 & 6,229 & $220(1.7) / 193(1.5)$ & $1.15(0.95-1.38)$ & 0.148 \\
\hline Angioedema $[16,17]$ & 2 & 5,844 & $7(0.06) / 5(0.04)$ & $1.40(0.44-4.42)$ & 0.565 \\
\hline Cough $[17,33]$ & 2 & 1,496 & $14(0.7) / 11(0.5)$ & $0.67(0.04-10.91)$ & 0.775 \\
\hline Hyperkalemia $[16,33]$ & 2 & 5,118 & $84(0.7) / 86(0.7)$ & $0.98(0.73-1.32)$ & 0.917 \\
\hline \multicolumn{6}{|l|}{$\begin{array}{l}\text { Severe AEs associated with BP } \\
\text { medication }\end{array}$} \\
\hline Hypotension $[16,33]$ & 2 & 5,118 & $17(0.14) / 3(0.02)$ & $2.19(0.03-164.77)$ & 0.723 \\
\hline Hyperkalemia [16,33] & 2 & 5,118 & $12(0.1) / 5(0.04)$ & $2.39(0.20-28.59)$ & 0.490 \\
\hline Renal failure $[16,33]$ & 2 & 5,118 & $35(0.3) / 40(0.3)$ & $1.47(0.26-8.23)$ & 0.658 \\
\hline Angioedema [16] & 1 & 4,733 & $6(0.05) / 4(0.04)$ & 1. $51(0.43-5.33)$ & 0.548 \\
\hline Syncope [16] & 1 & 4,733 & $12(0.1) / 5(0.04)$ & $2.41(0.85-6.83)$ & 0.088 \\
\hline Arrhythmia [16] & 1 & 4,733 & $12(0.1) / 3(0.03)$ & $4.02(1.13-14.21)$ & 0.020 \\
\hline
\end{tabular}

harm. In addition, the targets used in the most intensive BP control strategies were not associated with adverse cardiovascular outcomes or increased rates of death.

The findings from this overview are consistent with a recent analysis in patients with diabetes [37] but contrast with reports from some individual studies $[16,18]$ and a recent meta-analysis that have suggested no benefit from more intensive BP lowering regimens [15]. In both cases the most likely reason for this is the limited statistical power of the prior analyses. Few of the individual trials have recorded sufficient numbers of events and achieved large enough BP differences between randomized groups, to detect the most plausible effects of intensive BP control regimens on vascular outcomes. This is particularly so for the outcome of myocardial infarction, which is less strongly associated with BP than stroke, and therefore requires a much larger body of data to detect the anticipated effects. The prior much cited overview [15] had similar problems because the selective inclusion criteria, addressing a very narrow clinical question, meant that much applicable evidence was excluded. In this report, we approximately doubled the numbers of participants and events available for analysis, in large part because we were able to include new data from three large trials [16-18].

Key to interpreting the plausibility of the new findings presented here is an understanding of the broader clinical and epidemiological context. Associations observed in cohort studies and risk reductions seen in clinical trials of $\mathrm{BP}$ lowering versus control both provide indications of the magnitude of benefit that might be anticipated as a consequence of the $7.5 / 4.5-\mathrm{mmHg}$ difference in $\mathrm{BP}$ seen in the current set of trials. The very close concordance between the expected benefits and those observed in this metaanalysis provides strong support for the validity of the current findings and argues for their wider generalisability.

It is now widely acknowledged that the observational association of $\mathrm{BP}$ with risk is direct and continuous to levels of BP far below the usual definition of hypertension [1-3]. Reported J-curve associations, seen mostly amongst patients with established disease, are likely to be attributable mostly to "reverse causation" - low BP is caused by the disease (e.g., prior heart attack) [38] and is associated with an increased risk of a poor outcome, but is not in itself the cause of the poor outcome. A number of recent post hoc analyses of clinical trial datasets have reignited concerns about the possibility of a J-curve for coronary disease at achieved systolic BP levels below $120 \mathrm{mmHg}$ [39-41]. However, these analyses are nonrandomised in nature and need to be considered in light of the potential for confounding. The consistency of benefit at different baseline and achieved BP levels in this and other systematic reviews of all available evidence [4] suggests that confounding is indeed the reason for these observations.

We found evidence of benefit for clinically important microvascular outcomes with intensive BP lowering strategies. Specifically, the risk of ESKD was reduced by $11 \%$. Similarly, trials in people with diabetes showed evidence of a reduced incidence of microalbuminuria and a trend towards a reduced incidence of retinopathy. Taken together, these results provide substantial reassurance about the renal safety of intensive BP lowering and suggest benefit for microvascular outcomes is likely.

The present overview did not provide especially clear evidence about the effects of more intensive BP control on side effects because the quantity of available data was limited. Adverse events and serious adverse events were not increased overall, but an increased frequency of hypotension was observed. Of note, absolute rates of serious side effects appeared to be low and infrequently led to discontinuation of the intensive BP lowering strategy, although reporting of these events was suboptimal so some caution must be exercised in interpreting these results. These findings would suggest that lower targets for $\mathrm{BP}$ are likely to be achievable for many individuals and that there would be significant net benefit to population health if the strategy were widely implemented, although more precise data regarding the totality of adverse outcomes would be important in clarifying the remaining uncertainty in this regard.

This overview benefits from the rigorous methodology used, the homogeneity of the individual trial results summarized by the meta- 


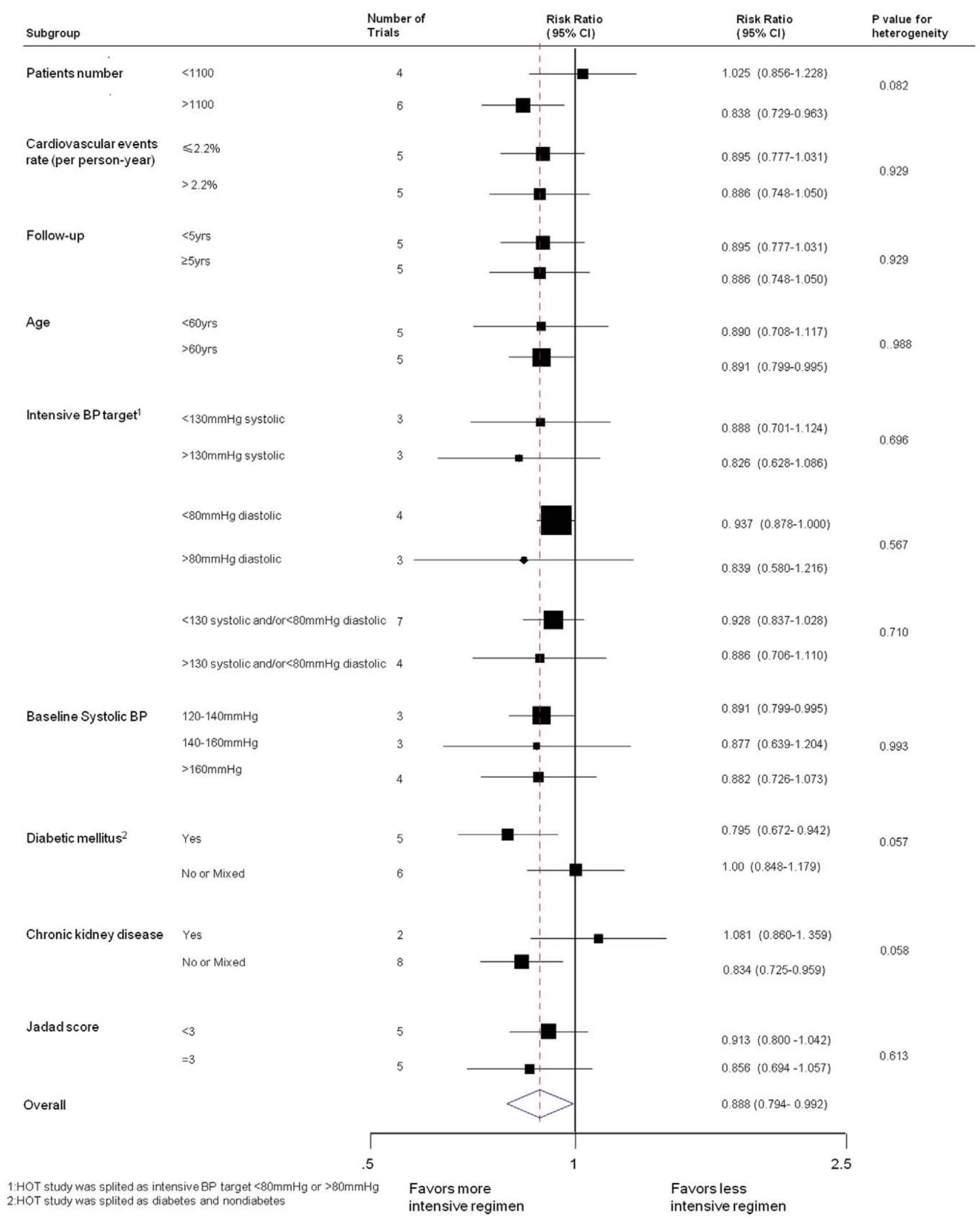

Figure 5. Effects of intensive BP lowering on the risk of major cardiovascular events in subgroups of trials. doi:10.1371/journal.pmed.1001293.g005 
Table 4. Univariate meta-regression of intensive blood pressure lowering on major cardiovascular outcomes.

\begin{tabular}{lllll}
\hline Variable & Studies & Scale & Proportional Change in RR (95\% Cl) & $p$-Value \\
\hline Patients & 10 & Per 100 & $1.0003(0.9981-1.0026)$ & 0.739 \\
Cardiovascular event rate (per person-year) & 10 & Per 1\% & $0.9224(0.7955-1.0694)$ & 0.243 \\
Follow-up & 10 & Per year & $1.0019(0.9405-1.0672)$ & 0.948 \\
Age & 10 & Per 10 y & $1.0058(0.7959-1.2710)$ & 0.956 \\
Baseline systolic BP & 10 & Per 1 mmHg & $0.9983(0.9865-1.0103)$ & 0.755 \\
\hline doi:10.1371/journal.pmed.1001293.t004 & & & &
\end{tabular}

analyses, and the consistent effects observed across a range of macro- and microvascular disease outcomes. All serve to provide reassurance about the likely validity of the primary conclusions. Chief among the limitations are the moderate number and size of trials available, the heterogeneity of participants in included trials, and in particular the few data to describe directly the effects of intensive BP lowering amongst individuals with uncomplicated hypertension. Most trials included in this study included participants with additional cardiovascular risk factors, including diabetes or CKD, which also limits the generalisability of the findings. Additionally, the subgroup analyses are based on the study characteristics rather than individual patient data (IPD). An IPD meta-analysis would provide important additional information. Finally, although this analysis suggests that BP targets at 130/80 or lower are likely to produce additional overall benefit, there is insufficient data to confirm a specific BP threshold. These analyses gain from the inclusion of analyses of renal outcomes. With ageing of the population, CKD is becoming an increasingly large cause of disease burden and documenting the effects on hard renal outcomes is central to estimating the overall balance of risks and benefits.

A range of research questions arise from this work, perhaps most importantly how best to achieve and maintain greater BP reductions in high-risk patients, particularly given the relatively modest BP differences between the randomized groups achieved on average in the completed trials. Large and rapid reductions may be less well tolerated, particularly if hypertension has been severe and longstanding, but the optimal ways to achieve this while maintaining adherence are still uncertain. It is apparent that lowdose combinations will be an important part of this solution [4244] but other approaches to improve treatment rates and adherence will be required.

In conclusion, these overviews provide support for clinical guidelines advocating more intensive BP lowering amongst highrisk patient groups, although the limitations of the available data mean that the results should be generalized with some caution. Whilst few large-scale randomized trials have been done to evaluate the effects of intensive BP lowering amongst patients with uncomplicated hypertension, and more data would clearly be helpful in defining the groups most likely to benefit as well as to suffer adverse effects, the totality of the current evidence suggests that benefits are likely to be greater than harms. BP lowering to below current thresholds may achieve additional benefits and

\section{References}

1. MacMahon S, Peto R, Cutler J, Collins R, Sorlie P, et al. (1990) Blood pressure, stroke, and coronary heart disease. Part 1, Prolonged differences in blood pressure: prospective observational studies corrected for the regression dilution bias. Lancet 335: 765-774

2. Lewington S, Clarke R, Qizilbash N, Peto R, Collins R (2002) Age-specific relevance of usual blood pressure to vascular mortality: a meta-analysis of reduce the burden of cardiovascular morbidity and mortality for many patients. If our data are applied to hypertensive patients at high cardiovascular risk with an annual cardiovascular event rate of about $2 \%$, the available data suggest that among every thousand such people, intensive BP lowering could prevent two of the 20 cardiovascular events expected to occur each year, while increasing one severe hypotension event.

\section{Supporting Information}

Figure S1 Begg's funnel plot for the assessment of publication bias in studies examining the effects of intensive BP lowering on major cardiovascular outcomes (Egger's test $p=0.668$ ), stroke $(p=0.125)$, myocardial infarction $(p=0.166)$, and end stage of kidney disease $(p=0.555)$.

(TIF)

Table S1 Quality analyses of the trials included in the systematic review and meta-analysis.

(DOCX)

Text S1 PRISMA checklist.

(DOCX)

Text S2 Search strategy.

(DOCX)

Text S3 Study protocol.

(DOG)

\section{Acknowledgments}

We are very grateful to Giuseppe Remuzzi and the REIN study group for providing useful additional information for this meta-analysis

\section{Author Contributions}

Conceived and designed the experiments: JL VP. Performed the experiments: JL PE. Analyzed the data: JL BN PE VP. Wrote the first draft of the manuscript: JL. Contributed to the writing of the manuscript: JL BN PE VP. ICMJE criteria for authorship read and met: JL BN PE TN MW AR HW SM FT GH JC VP. Agree with manuscript results and conclusions: JL BN PE TN MW AR HW SM FT GH JC VP. Contributed to data interpretation and critical revision of the report: TN MW AR HW SM FT GH JC.

individual data for one million adults in 61 prospective studies. Lancet 360: 1903-1913.

3. Lawes CM, Rodgers A, Bennett DA, Parag V, Suh I, et al. (2003) Blood pressure and cardiovascular disease in the Asia Pacific region. J Hypertens 21: 707-716.

4. Law MR, Morris JK, Wald NJ (2009) Use of blood pressure lowering drugs in the prevention of cardiovascular disease: meta-analysis of 147 randomised trials 
in the context of expectations from prospective epidemiological studies. BMJ 338: b1665.

5. Turnbull F (2003) Effects of different blood-pressure-lowering regimens on major cardiovascular events: results of prospectively-designed overviews of randomised trials. Lancet 362: 1527-1535.

6. Mancia G, De Backer G, Dominiczak A, Gifkova R, Fagard R, et al. (2007) 2007 Guidelines for the Management of Arterial Hypertension: The Task Force for the Management of Arterial Hypertension of the European Society of Hypertension (ESH) and of the European Society of Cardiology (ESC). J Hypertens 25: 1105-1187.

7. (2004) K/DOQI clinical practice guidelines on hypertension and antihypertensive agents in chronic kidney disease. Am J Kidney Dis 43: S1-290.

8. Williams B, Poulter NR, Brown MJ, Davis M, McInnes GT, et al. (2004) British Hypertension Society guidelines for hypertension management 2004 (BHS-IV): summary. BMJ 328: 634-640.

9. Chobanian AV, Bakris GL, Black HR, Cushman WC, Green LA, et al. (2003) The Seventh Report of the Joint National Committee on Prevention, Detection, Evaluation, and Treatment of High Blood Pressure: the JNC 7 report. JAMA 289: 2560-2572

10. Whitworth JA (2003) 2003 World Health Organization (WHO)/International Society of Hypertension (ISH) statement on management of hypertension. J Hypertens 21: 1983-1992.

11. Mancia G, Laurent S, Agabiti-Rosei E, Ambrosioni E, Burnier M, et al. (2009) Reappraisal of European guidelines on hypertension management: a European Society of Hypertension Task Force document. J Hypertens 27: 2121-2158.

12. Ogihara T, Kikuchi K, Matsuoka H, Fujita T, Higaki J, et al. (2009) The Japanese Society of Hypertension Guidelines for the Management of Hypertension (JSH 2009). Hypertens Res 32: 3-107.

13. hypertension WgoCgftmo (2011) 2010 Chinese guidelines for the management of hypertension. Chin J Hypertens 19: 701-743.

14. Excellence NIfHaC (2011) Hypertension: clinical management of primary hypertension in adults. CG127. Available: http://guidanceniceorguk/CG127/ Guidance/pdf/English

15. Arguedas JA, Perez MI, Wright JM (2009) Treatment blood pressure targets for hypertension. Cochrane Database Syst Rev: CD004349.

16. Cushman WC, Evans GW, Byington RP, Goff DC, Jr., Grimm RH, Jr., et al. (2010) Effects of intensive blood-pressure control in type 2 diabetes mellitus. N Engl J Med 362: 1575-1585.

17. Verdecchia P, Staessen JA, Angeli F, de Simone G, Achilli A, et al. (2009) Usual versus tight control of systolic blood pressure in non-diabetic patients with hypertension (Cardio-Sis): an open-label randomised trial. Lancet 374: 525-533.

18. Ogihara T, Saruta T, Rakugi H, Matsuoka H, Shimamoto K, et al. (2010) Target blood pressure for treatment of isolated systolic hypertension in the elderly: valsartan in elderly isolated systolic hypertension study. Hypertension 56: $196-202$

19. Liberati A, Altman DG, Tetzlaff J, Mulrow C, Gotzsche PC, et al. (2009) The PRISMA statement for reporting systematic reviews and meta-analyses of studies that evaluate health care interventions: explanation and elaboration. Ann Intern Med 151: W65-94.

20. Woodward M (2005) Epidemiology: design and data analysis. 2nd edition. Boca Raton (Florida): Chapman and Hall/CRC Press.

21. Egger M, Davey Smith G, Schneider M, Minder C (1997) Bias in meta-analysis detected by a simple, graphical test. BMJ 315: 629-634.

22. Toto RD, Mitchell HC, Smith RD, Lee HC, McIntire D, et al. (1995) "Strict" blood pressure control and progression of renal disease in hypertensive nephrosclerosis. Kidney Int 48: 851-859.

23. (1998) Tight blood pressure control and risk of macrovascular and microvascular complications in type 2 diabetes: UKPDS 38. UK Prospective Diabetes Study Group. BMJ 317: 703-713.

24. Hansson L, Zanchetti A, Carruthers SG, Dahlof B, Elmfeldt D, et al. (1998) Effects of intensive blood-pressure lowering and low-dose aspirin in patients with hypertension: principal results of the Hypertension Optimal Treatment (HOT) randomised trial. HOT Study Group. Lancet 351: 1755-1762.
25. Estacio RO, Jeffers BW, Gifford N, Schrier RW (2000) Effect of blood pressure control on diabetic microvascular complications in patients with hypertension and type 2 diabetes. Diabetes Care 23 Suppl 2: B54-64.

26. Schrier RW, Estacio RO, Esler A, Mehler P (2002) Effects of aggressive blood pressure control in normotensive type 2 diabetic patients on albuminuria, retinopathy and strokes. Kidney Int 61: 1086-1097.

27. Wright JT, Jr., Bakris G, Greene T, Agodoa LY, Appel LJ, et al. (2002) Effect of blood pressure lowering and antihypertensive drug class on progression of hypertensive kidney disease: results from the AASK trial. JAMA 288: 2421-2431.

28. Klahr S, Levey AS, Beck GJ, Cagoiula AW, Hunsicker L, et al (1994) The effects of dietary protein restriction and blood-pressure control on the progression of chronic renal disease. Modification of Diet in Renal Disease Study Group. N Engl J Med 330: 877-884.

29. Sarnak MJ, Greene T, Wang X, Beck G, Kusek JW, et al. (2005) The effect of a lower target blood pressure on the progression of kidney disease: long-term follow-up of the modification of diet in renal disease study. Ann Intern Med 142: 342-351.

30. Ruggenenti P, Perna A, Loriga G, Ganeva M, Ene-Iordache B, et al. (2005) Blood-pressure control for renoprotection in patients with non-diabetic chronic renal disease (REIN-2): multicentre, randomised controlled trial. Lancet 365: 939-946.

31. Estacio RO, Coll JR, Tran ZV, Schrier RW (2006) Effect of intensive blood pressure control with valsartan on urinary albumin excretion in normotensive patients with type 2 diabetes. Am J Hypertens 19: 1241-1248.

32. (2008) Principal results of the Japanese trial to assess optimal systolic blood pressure in elderly hypertensive patients (JATOS). Hypertens Res 31: 21152127.

33. Wuhl E, Trivelli A, Picca S, Litwin M, Peco-Antic A, et al. (2009) Strict bloodpressure control and progression of renal failure in children. N Engl J Med 361: $1639-1650$

34. Schrier R, McFann K, Johnson A, Chapman A, Edelstein C, et al. (2002) Cardiac and renal effects of standard versus rigorous blood pressure control in autosomal-dominant polycystic kidney disease: results of a seven-year prospective randomized study. J Am Soc Nephrol 13: 1733-1739.

35. Appel LJ, Wright JT, Jr., Greene T, Agodoa LY, Astor BC, et al. (2010) Intensive blood-pressure control in hypertensive chronic kidney disease. N Engl J Med 363: 918-929.

36. Hayashi K, Saruta T, Goto Y, Ishii M (2010) Impact of renal function on cardiovascular events in elderly hypertensive patients treated with efonidipine. Hypertens Res 33: 1211-1220.

37. Reboldi G, Gentile G, Angeli F, Ambrosio G, Mancia G, et al. (2011) Effects of intensive blood pressure reduction on myocardial infarction and stroke in diabetes: a meta-analysis in 73,913 patients. J Hypertens 29: 1253-1269.

38. Flack JM, Neaton J, Grimm R, Jr., Shih J, Cutler J, et al. (1995) Blood pressure and mortality among men with prior myocardial infarction. Multiple Risk Factor Intervention Trial Research Group. Circulation 92: 2437-2445.

39. Berl T, Hunsicker LG, Lewis JB, Pfeffer MA, Porush JG, et al. (2005) Impact of achieved blood pressure on cardiovascular outcomes in the Irbesartan Diabetic Nephropathy Trial. J Am Soc Nephrol 16: 2170-2179.

40. Messerli FH, Mancia G, Conti CR, Hewkin AC, Kupfer S, et al. (2006) Dogma disputed: can aggressively lowering blood pressure in hypertensive patients with coronary artery disease be dangerous? Ann Intern Med 144: $884-893$.

41. Sleight P, Redon J, Verdecchia P, Mancia G, Gao P, et al. (2009) Prognostic value of blood pressure in patients with high vascular risk in the Ongoing Telmisartan Alone and in combination with Ramipril Global Endpoint Trial study. J Hypertens 27: 1360-1369.

42. Wald DS, Law M, Morris JK, Bestwick JP, Wald NJ (2009) Combination therapy versus monotherapy in reducing blood pressure: meta-analysis on 11,000 participants from 42 trials. Am J Med 122: 290-300.

43. Law MR, Wald NJ, Morris JK, Jordan RE (2003) Value of low dose combination treatment with blood pressure lowering drugs: analysis of 354 randomised trials. BMJ 326: 1427.

44. Feldman RD, Zou GY, Vandervoort MK, Wong CJ, Nelson SA, et al. (2009) A simplified approach to the treatment of uncomplicated hypertension: a cluster randomized, controlled trial. Hypertension 53: 646-653. 


\section{Editors' Summary}

Background. About a third of US and UK adults have high blood pressure (hypertension). Although hypertension has no obvious symptoms, it can lead to heart attacks, stroke, and other forms of cardiovascular disease, to kidney failure, and to retinopathy (blindness caused by damage to the blood vessels in the back of the eye). Hypertension is diagnosed by measuring blood pressure (BP)-the force that blood moving around the body exerts on the inside of large blood vessels. $B P$ is highest when the heart is pumping out blood (systolic BP) and lowest when it is refilling with blood (diastolic BP). A normal adult BP is defined as a systolic BP of less than 130 millimeters of mercury $(\mathrm{mmHg})$ and a diastolic BP of less than $85 \mathrm{mmHg}$ (a BP of 130/85). A reading of more than $140 / 90$ indicates hypertension. Many factors affect BP, but overweight people and individuals who eat fatty or salty food are at high risk of developing hypertension. Mild hypertension can be corrected by making lifestyle changes, but people often take antihypertensive drugs to reduce their BP.

Why Was This Study Done? Doctors usually try to reduce the BP of their hypertensive patients to $140 / 90 \mathrm{mmHg}$. However, some treatment guidelines now advocate a target BP of $130 / 80 \mathrm{mmHg}$ for individuals at high risk of lifethreatening cardiovascular events, such as people with diabetes or kidney impairment. But does more intensive BP lowering actually reduce the risk of heart attacks and stroke? Although placebo-controlled randomized trials of BP lowering have suggested that a $10 \mathrm{mmHg}$ fall in systolic BP is associated with a $22 \%$ reduction in the risk in coronary heart disease and a $41 \%$ reduction in the risk of stroke, it is unclear whether intensive BP lowering strategies are associated with greater reductions in the risk of cardiovascular disease than standard strategies. In this systematic review (a search that uses predefined criteria to identify all the research on a given topic) and meta-analysis (a statistical method for combining the results of studies), the researchers investigate the effects of intensive BP lowering on cardiovascular, eye, and renal outcomes.

What Did the Researchers Do and Find? The researchers identified 15 randomized controlled trials in which more than 37,000 participants were randomly assigned to antihypertensive drug-based strategies designed to achieve different target BPs. On average, the more intensive strategies reduced the $\mathrm{BP}$ of participants by $7.5 / 4.5 \mathrm{mmHg}$ more than the less intensive strategies. Compared to standard BP lowering strategies, more intensive BP lowering strategies reduced the risk of major cardiovascular events (a composite endpoint comprising heart attack, stroke, heart failure, and cardiovascular death) by $11 \%$, the risk of heart attack by $13 \%$, the risk of stroke by $24 \%$, the risk of end-stage kidney disease by $11 \%$, and the risk of albuminuria (protein in the urine, a sign of kidney damage) by $10 \%$. There was also a trend towards a reduced risk for retinopathy with more intensive BP lowering but no clear reduction in cardiovascular or noncardiovascular deaths. Finally, aiming for a lower BP target did not increase the rate of drug discontinuation or the risk of serious adverse events apart from hypotension (very low BP).

What Do These Findings Mean? These findings suggest that, although intensive BP lowering regimens have no clear effect on the risk of death, they may provide greater protection against cardiovascular events than standard BP lowering regimens. Indeed, the researchers calculate that among every thousand hypertensive patients with a high cardiovascular risk, more intensive BP lowering could prevent two of the 20 cardiovascular events expected to happen every year. Although intensive BP lowering did not seem to increase the risk of severe adverse effects, the accuracy of this finding is limited by inconsistent reporting of adverse events in the trials included in this study. Moreover, because most of the trial participants had additional risk factors for cardiovascular events such as diabetes and chronic kidney disease, these findings may not be generalizable to people with hypertension alone. Thus, although this study suggests that a target BP of $130 / 80$ is likely to produce an additional overall benefit compared to a target of 140/90, more trials are needed to confirm this conclusion and to determine the best way to reach the lower target.

Additional Information. Please access these websites via the online version of this summary at http://dx.doi.org/10. 1371/journal.pmed.1001293.

- The US National Heart Lung and Blood Institute has patient information about high blood pressure (in English and Spanish)

- The American Heart Association provides information on high blood pressure and on cardiovascular diseases (in several languages); it also provides personal stories from people dealing with high blood pressure

- The UK National Health Service (NHS) Choices website also provides detailed information for patients about hypertension, cardiovascular disease and kidney disease; the NHS Local website has a collection of personal stories about hypertension and a series of films that explain hypertension

- MedlinePlus provides links to further information about high blood pressure, heart disease, stroke, and kidney disease (in English and Spanish) 